Int. J. Dev. Biol. 57: 731-739 (2013)

doi: $10.1387 / \mathrm{ijdb} .120189 \mathrm{me}$

\title{
Ontogenetic consequences of dysgenic crosses in Drosophila virilis
}

\author{
MARINA I. SOKOLOVA 1 ,\#, ELENA S. ZELENTSOVA ${ }^{1, \#, ~ N A T A L I A ~ G . ~ S H O S T A K ~}{ }^{1}$, \\ NIKOLAY V. ROZHKOV ${ }^{3}$ and MICHAEL B. EVGEN'EV*,1,2 \\ ${ }^{1}$ Engelhardt Institute of Molecular Biology, Moscow, Russia, ${ }^{2}$ Institute of Cell Biophysics, RAS, Pushchino, Moscow \\ region, Russia and ${ }^{3}$ Cold Spring Harbor Laboratory, Cold Spring Harbor, New York, USA
}

\begin{abstract}
Hybrid dysgenesis (HD) syndrome in Drosophila virilis presumably results from the mobilization of several unrelated mobile genetic elements in dysgenic hybrids. Morphogenetic events during oogenesis and spermatogenesis were investigated in detail in the progeny of $D$. virilis dysgenic crosses. Using germ-cell specific anti-Vasa staining, we monitored the fate of germline cells at different ontogenetic stages in strains of $D$. virilis and their hybrids. Anti-Vasa staining indicated that the major loss of pole cells occurs in dysgenic embryos at stage 11-14 after primordial germ cells (PGC) pass the midgut wall. At later ontogenetic stages, including larvae, pupae and imagoes, we often observed an abnormal development of gonads in dysgenic individuals with a frequent occurrence of unilateral and bilateral gonadal atrophy. Dysgenic females were characterized by the presence of various sterile ovarian phenotypes that predominantly include agametic ovarioles, while other atypical forms such as tumor-like ovarioles and dorsalized ovariolar follicles may also be present. Testis abnormalities were also frequently observed in dysgenic males. The sterility manifestations depended on the strain, the growing temperature and the age of the flies used in crosses. The observed gonadal sterility and other HD manifestations correlated with the absence of maternal piRNAs homologous to Penelope and other transposons in the early dysgenic embryos. We speculate that gonadal abnormalities mimicking several known sterility mutations probably result from the disturbance of developmental gene expression machinery due to the activation of unrelated families of transposons in early dysgenic embryos.
\end{abstract}

KEY WORDS: Drosophila virilis, anti-Vasa staining, sterility, hybrid dysgenesis, transposon

\section{Introduction}

The deleterious traits that occur in the interstrain hybrids of Drosophila melanogaster, described as hybrid dysgenesis (HD) by M. Kidwell (1983), were later also found in Drosophila virilis (Lozovskaya et al., 1990; Scheinkeret al., 1990). Among the dysgenic traits, such as male recombination, point mutations, chromosomal aberrations and nondisjunction, the most prominent and easily visualized trait in the HD systems is gonadal atrophy in adult flies (Kidwell 1983; Lozovskaya et al., 1990; Evgen'evet al. 1997; Blumenstiel and Hartl, 2005).

Gonadal sterility in $D$. melanogaster HD manifests in two independent systems known as the $I-R$ and $P-M$ systems, which are controlled by the $I$ and $P$ elements, respectively (for excellent background material, see Bucheton et al.;2002, Rio, 2002; Kidwell and Lisch 2002; and Khurana et al., 2011).

However, in $D$. virilis, several unrelated transposable elements (TEs) belonging to different classes (Penelope, Ulysses, Paris, Helena, Telemac, and likely Tv1) are simultaneously activated (Scheinker et al., 1990; Petrov et al., 1995; Evgen'ev et al., 1997; Vieira et al., 1998). It is noteworthy that recently mobilization of several resident TEs besides $P$-elements has been also demonstrated in the P-M dysgenic hybrids (Khurana et al., 2011).

It was suggested that Penelope and probably Paris and Helena

Abbreviations used in this paper: GD, gonadal dysgenesis; HD, hybrid dysgenesis; PGC, primordial germ cell; TE, transposable element.

\footnotetext{
*Address correspondence to: Michael B. Evgen'ev, Engelhardt Institute of Molecular Biology, Vavilov str., 32, Moscow, GSP-1, 119991 Russia.

E-mail: misha672011@yahoo.com \#Note: These authors contributed equally to this work.
}

Supplementary Material (5 figures and 3 tables) for this paper is available at: http://dx.doi.org/10.1387/ijdb.120189me

Accepted: 27 May 2013. Final, author-corrected PDF published online: 20 November 2013.

ISSN: Online 1696-3547, Print 0214-6282 
play important roles in HD manifestations because the $D$. virilis strains used to produce dysgenic phenotypes differ in the presence of these TEs (Petrov et al., 1995; Evgen'ev et al., 1997; Vieira et al., 1998). In general, gonadal sterility and other HD manifestations observed in $D$. virilis dysgenic males and females are reminiscent of those observed in the $D$. melanogaster $P-M H D$ system. In the adult hybrids resulting from crosses between females lacking functional $P$-elements, which encode active transposase from certain laboratory strains and males carrying complete $P$-elements from wild populations (or the equivalent), gonadal sterility manifests as rudimentary ovaries with partially or completely sterile sets of ovarioles and rudimentary testes depending on the temperature conditions. Sterility in this system is more frequent in females than in males (Kidwell et al., 1983). The l-factor in I- $R$ HD system is responsible for another type of fly sterility resulting from embryo mortality, and the transposition of the l-element is sex specific because it never occurs in males (Finnegan, 1989; Bucheton et al., 2002). Reciprocal crosses in either system result in essentially fertile progeny.

In the $P$-M dysgenic embryos, germline cells degenerate autonomously and independently of the surrounding somatic tissue, including ovarian mesodermal tissue. This degeneration results in agametic sterile ovarioles in adult females (Niki, 1986).

It has also been shown that the third intron of the $P$ element is spliced normally only in the primordial embryonic germ cells (pole cells) and adult germline cells (Laski and Rubin, 1989). This mechanism is crucial for encoding an active transposase, which functions to excise or insert $P$-elements in $D$. melanogaster germline cells. Notably, the Penelope retroelement in $D$. virilis also contains an intron and may produce spliced and unspliced mRNAs; however, the role of this intron splicing in Penelopefunction and HD is unknown (Arkhipova et al., 2003; Shostak et al., 2008).

The strains used in the $D$. virilis dysgenic crosses by analogy to the previously described $D$. melanogaster $P$-Msystem are designated as " $P$-like" (such strains contain multiple full-length Penelope copies) and "M-like" (such strains lack intact Penelopecopies in the genome). All dysgenic traits are the result of crosses between the males from an old $D$. virilis laboratory marked strain 160 (strong $P$-like strain) possessing multiple copies of Penelope and the females of various wild-type strains lacking active Penelope elements (Lozovskaya et al., 1990; Vieira et al., 1998; Blumenstiel and Hartl, 2005; Rozhkov et al., 2010; Rozhkov et al., 2011).

The parental $D$. virilis $P$-like strain itself may exhibit a significant level of sterility as observed, for example, in the $D$. melanogaster Harwich $P$-like strain (Lozovskaya et al., 1990). In both HD systems, bilateral and unilateral ovary and testis sterility are often observed. Visible chromosomal mutations and other features in the progeny of $D$. virilis dysgenic crosses are also partially shared with the P-M HD system described in D. melanogaster (Lozovskaya et al., 1990; Evgen'ev et al., 1997; Vieira et al., 1998). When we previously discovered HD in $D$. virilis, we estimated the total frequency of gonadal sterility in the adult progeny from dysgenic and reciprocal crosses (Lozovskaya et al., 1990). However, at that time, we did not describe the

pattern of the observed gonad abnormalities or the ontogenetic stages of occurrence.

Subsequently, Blumenstiel and Hartl (2005) attempted to find a correlation between maternally transmitted Penelope-derived small RNAs and the sterility phenotypes observed in the adult $D$. virilis dysgenic hybrids, similar to what was clearly demonstrated for the $P-M$ and $I-R$ dysgenesis systems in $D$. melanogaster (Brennecke et al., 2008; Chambeyron et al., 2008; Khurana et al., 2011). Here, we continue our studies on $D$. virilis $\mathrm{HD}$ and provide a detailed analysis of the gonad abnormalities observed in the ontogenesis of dysgenic hybrids. Using a germ cell-specific Vasa-antibody, we determined the stages where primordial germ cell death begins in dysgenic embryos and monitored the consequences of abnormal primary gonad formation in different stages of morphogenesis, including imagoes.

The observed dysgenic phenotypes correlated with the expression of Penelope and several other TEs in the dysgenic embryos and were inversely correlated with the levels of maternally supplied primary piRNAs homologous to these TEs.

\section{Results}

\section{Frequency and spectrum of gonadal abnormalities observed in dysgenic females}

Each pair of the $D$. virilis ovaries consists of $63-65$ ovarioles with an anterior structure called germarium. Similar to $D$. melanogaster, the germarium harbors 2-3 germ stem cells (GSC) in its anteriormost region (GSC niche), two follicle stem cells (FSC)
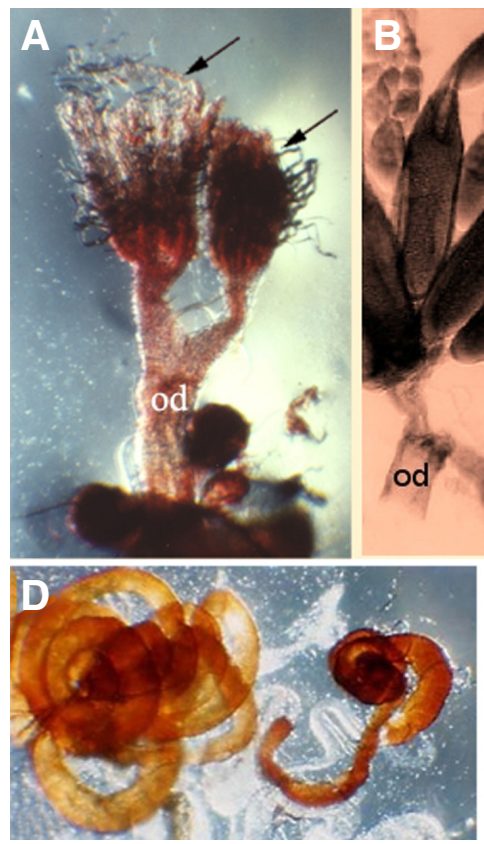

Fig. 1. Ovaries and testes of dysgenic progeny of $D$. virilis (A) and (B) (strain 9 female x strain 160 male) and $D$. melanogaster (C) (female $D f(1), y w^{67 c 23(2)} \times$ male Harwich) grown at $2^{\circ} \mathbf{C}$. (A) Bilateral sterile ovaries; (B) left normal ovary and right sterile atrophied ovary; (C) unilateral sterile ovary; od-oviduct; nf-normal follicle developed to an egg; arrows indicate atrophied ovaries; (A,C) neutral red, (B) unstained. 20x.(D) The right testis is reduced; the left testis has a normal size. (E) Bilateral sterile darkly pigmented testes are shown. (F) A cistern-like abnormal testis surrounded by developed spermatozoids released from the damaged testis. 
TABLE 1

\section{THE FREQUENCY (IN \%) OF DIFFERENT TYPES OF OVARIES IN THE FEMALES OF STRAINS AND F1 CROSSES (FEMALES X MALES)}

\begin{tabular}{cccccccc}
$\begin{array}{c}\text { Strains and } \\
\text { crosses }\end{array}$ & $\begin{array}{c}\text { No. of } \\
\text { females }\end{array}$ & $\mathbf{N} / \mathbf{N}$ & $\mathbf{N} / \mathbf{S N}$ & $\mathbf{N} / \mathbf{S T}$ & $\mathbf{S N} / \mathbf{S N}$ & $\mathbf{S N} / \mathbf{S T}$ & $\mathbf{S T} / \mathbf{S T}$ \\
\hline 9 & 106 & $100(106)$ & 0 & 0 & 0 & 0 & 0 \\
$9 \times 160$ & 442 & $49(219)$ & $10(42)$ & $12(55)$ & $7(30)$ & $8(36)$ & $14(62)$ \\
$160 \times 9$ & 100 & $98(98)$ & 0 & $1(1)$ & 0 & 0 & $1(1)$ \\
160 & 112 & $97(109)$ & 0 & $1(1)$ & $1(1)$ & 0 & $1(1)$ \\
13 & 181 & $100(181)$ & 0 & 0 & 0 & 0 & 0 \\
$13 \times 160$ & 271 & $23(62)$ & $10(26)$ & $11(31)$ & $11(30)$ & $22(22)$ & $23(62)$ \\
$160 \times 13$ & 139 & $97(135)$ & 0 & $1(2)$ & 0 & $1(1)$ & $1(1)$ \\
1 & 32 & $97(31)$ & 0 & $3(1)$ & 0 & 0 & 0 \\
$1 \times 160$ & 60 & $84(50)$ & $2(1)$ & $5(3)$ & $2(1)$ & $2(1)$ & $5(3)$ \\
142 & 24 & $42(10)$ & $4(1)$ & $4(1)$ & $50(12)$ & 0 & 0 \\
$142 \times 160$ & 48 & $52(25)$ & $19(9)$ & $13(6)$ & $8(4)$ & $2(1)$ & $6(3)$ \\
$160 \times 142$ & 69 & $98(67)$ & 0 & $1(1)$ & 0 & 0 & $1(1)$ \\
110 & 36 & $100(36)$ & 0 & 0 & 0 & 0 & 0 \\
$110 \times 160$ & 112 & $97(109)$ & 0 & 0 & 0 & 0 & $3(3)$ \\
$160 \times 110$ & 91 & $95(86)$ & 0 & 0 & $1(1)$ & 0 & $4(4)$ \\
$142 \times 110$ & 78 & $94(73)$ & 0 & $1(1)$ & $2(2)$ & 0 & $1(1)$ \\
$110 \times 142$ & 58 & $100(58)$ & 0 & 0 & 0 & 0 & 0 \\
\hline
\end{tabular}

$\mathrm{N} / \mathrm{N}$ - both normal ovaries, N/SN* - one normal and another semi-sterile ovary, N/ST - normal/ sterile, SN/SN - both semi-sterile, SN/ST - semi-sterile/sterile, ST/ST - both sterile. The numbers in brackets indicate the number of females with the certain type of ovaries.

The normal ovarian phenotype was indicated as "N", indicating that all or more than $3 / 4$ of ovarioles in the ovary did not deviate from the normal developmental pattern.

${ }^{*} \mathrm{~A}$ quite frequent ovarian abnormal phenotype observed in certain $D$. virilis strains and hybrids, designated by us as semi-normal (SN), manifested as a set of different types of ovarioles coexisting in the same ovary, including agametic and tumor-like ovarioles.

and their differentiated daughters, cystoblasts and prefollicle cells, respectively (Spradling, 1993).

To estimate the total frequency of sterility, we counted flies (both males and females) with complete unilateral or bilateral gonadal atrophy in a sample of $D$. virilis strains and their hybrids produced in bidirectional crosses with the "classical" $P$-like strain 160 (Table 1). Besides, we estimated the frequency of various abnormal ovarioles within each individual. It was evident, as has been previously demonstrated (Lozovskaya et. al, 1990; Evgen'ev et al., 1997; Blumenstiel and Hartl, 2005), that significant levels of gonadal sterility with complete bilateral or unilateral gonadal atrophy were observed in the dysgenic progeny of crosses between females from typical $M$-like strains lacking Penelope (e.g., strain 9) and males of strain 160 (Figs. 1A,B). It is noteworthy that "agametic" rudimentary ovarioles with no obvious separation between the egg chambers, that represent the major manifestation of gonadal sterility in $D$. virilis dysgenic females, phenotypically resembled those observed in $P$-M hybrid dysgenesis in $D$. melanogaster (Fig. 1C).

Ovaries of the wild-type M-like strain 9 have approximately 6365 ovarioles, none of which deviate from the standard, whereas ovaries from other $M$-like and $P$-like strains had sets of ovarioles of different numbers and phenotypes (Table 2), for example, 36-37 ovarioles in the progeny of the cross between females of strain 13 and males of strain 160. Moreover, a bilaterally normal phenotype $(\mathrm{N} / \mathrm{N})$ in $100 \%$ of females was found in strains 9,13 and 110 (Table 1), but different abnormal ovariolar phenotypes (predominant "agametic" and "tumor-like") leading to complete or partial ovarian sterility were detected in dysgenic hybrids (Table 2 and Supp. Fig. S1).

To further characterize the developmental abnormalities of dysgenic ovaries we took advantage of antibodies that specifically label germline cells and fusomes within the germ cells, anti-Vasa and anti-1B1 respectively (see Huynh, 2005 for excellent review). Agametic ovarioles often produced in dysgenic hybrids lack normal germ cells as revealed by staining with anti-Vasa and 1B1 antibodies and appeared as empty tubes either lacking stained material or filled with small cells or degraded cell conglomerates (Supp. Fig. S1). In rare cases DAPI staining revealed the presence of mesodermal cells, follicle epithelial cells along the tube and, occasionally, a few large, presumably cap cells adjacent to the filaments (data not shown). "Tumor-like"ovarioles lacked the true germarium zone as well as true follicles (Supp. Fig. S1 C,F). This type of abnormality was rather frequent in the dysgenic progeny between strains 13 and 160 (Table 2). DAPI staining of "tumor-like"ovarioles revealed small follicle epithelium cells, a band of stem prefollicular cells, and a cavity in which a few large, presumably undifferentiated germline cells were sometimes observed in an unattached state (Supp. Fig. $\mathrm{S} 1 \mathrm{G})$. The sterile phenotype, in its most extreme bilateral or unilateral manifestation with atrophied ovarioles or with the addition of a few tumor-like ovarioles, reached high levels in the crosses between M-like strains and strain 160 (Table 1 and 2).

In addition to the severe defects in oogenesis, the dysgenic hybrids showed a range of other phenotypes that occurred at lower penetrance. These phenotypes included additional spermathecae and follicles with disturbed polarity. The swallowed, inverted (turned $180^{\circ} \mathrm{degrees}$ with respect to each other) mature follicles were sometimes observed with abnormal appendages. The frequency of females with such abnormal follicles was roughly similar to the frequency of females with tumor-like ovarioles, comprising $2-4 \%$, depending on the strain (Table 2).

Importantly, the levels of gonadal atrophy observed in the progeny of dysgenic crosses significantly decreased with the age of parents used in crosses and increased in the flies grown at low $\left(18^{\circ} \mathrm{C}\right)$ temperatures (Tables S2 and S3). On the other hand the

TABLE 2

\section{THE FREQUENCY (IN \%) OF FEMALES THAT CONTAIN DIFFERENT OVARIOLAR PHENOTYPES IN THE STRAINS, DYSGENIC AND RECIPROCAL HYBRIDS OF DROSOPHILA VIRILIS}

\begin{tabular}{ccccc} 
Strains and crosses & Agameticovarioles & $\begin{array}{c}\text { Tumor-like } \\
\text { ovarioles }\end{array}$ & $\begin{array}{c}\text { Abnormal } \\
\text { polarity }\end{array}$ & $\begin{array}{c}\text { Abnormal } \\
\text { appendages }\end{array}$ \\
\hline 9 & 1 & 0 & 0 & 0 \\
160 & 9 & 0 & 1 & 2 \\
$9 \times 160$ & $98^{*}$ & 4 & 2 & 1 \\
$160 \times 9$ & $95^{*}$ & 0 & 0 & 0 \\
13 & 91 & 2 & 13 & 0 \\
$13 \times 160$ & 97 & 53 & 2 & 0 \\
$160 \times 13$ & 98 & 3 & 0 & 0 \\
$1 \times 160$ & 1 & 3 & 3 & 0 \\
142 & 1 & 0 & 12 & 4 \\
$142 \times 160$ & 1 & 8 & 4 & 0 \\
$160 \times 142$ & 0 & 0 & 0 & 0 \\
110 & 1 & 0 & 0 & 0 \\
$160 \times 110$ & 1 & 0 & 0 & 0 \\
$9 \times 110$ & 1 & 2 & 0 & 0
\end{tabular}

The numbers of ovaries with various types of ovariolar abnormalities were determined $(n=100$ females were included for each entity; three replicates were performed with $n=30-35$ females).

*We counted all females that have at least one or several agameticovarioles in otherwise normal ovary and, hence, we detected similar numbers of females with agameticovarioles in dysgenic and reciprocal hybrids although the hybrids from such crosses differ drastically in the levels of gonadal atrophy (sterility) as seen in Table 1. 
aged dysgenic males and females are not becoming less sterile (Table S2), as was shown for HD in D. melanogaster (Bucheton et al., 2002; Khurana et al., 2011).

\section{High levels of sterility and gross abnormalities of dysgenic testes}

The more extreme phenotype of testis atrophy was often accompanied by heavy pigmentation and was manifested in a low number or a complete lack of spermatocysts (Fig. 1 D,E and Table 3). Cistern-like testes filled with spermatocytes represent another type of abnormal gonad development, and such abnormal testes were sometimes found unconnected to ectodermal ejaculatory ducts (Fig. 1 F). When studying testis phenotypes, we distinguished, similar to the ovarian phenotypes, normal (N), sterile (ST) with severely reduced testis, and semi-normal (SN) types of testes. All of these phenotypes had unilateral or bilateral manifestations (Table 3). As in the case of ovaries, there were different frequencies of testis phenotypes in different experiments, and the maximal level of male sterility was observed in dysgenic hybrids under low temperature conditions $\left(18^{\circ} \mathrm{C}\right.$; Table S3). Overall, the frequency of female gonad abnormalities in dysgenic hybrids was significantly higher than that of the males (Tables S2 and S3), however, this value greatly depends on the strains used in dysgenic crosses (Tables 1 and 3).

\section{Severely reduced number of pole cells constituting the primary gonads of dysgenic embryos}

The vasa gene (vas) is essential for germline development in Drosophila melanogaster and other Drosophila species, including D. virilis (Lasko and Ashburner, 1990). Zygotic vasa is expressed in pole cells earlier than any other pole cell-expressing genes that have thus far been identified. Moreover, the Vasa protein is highly conserved and is continuously present in Drosophila germline cells
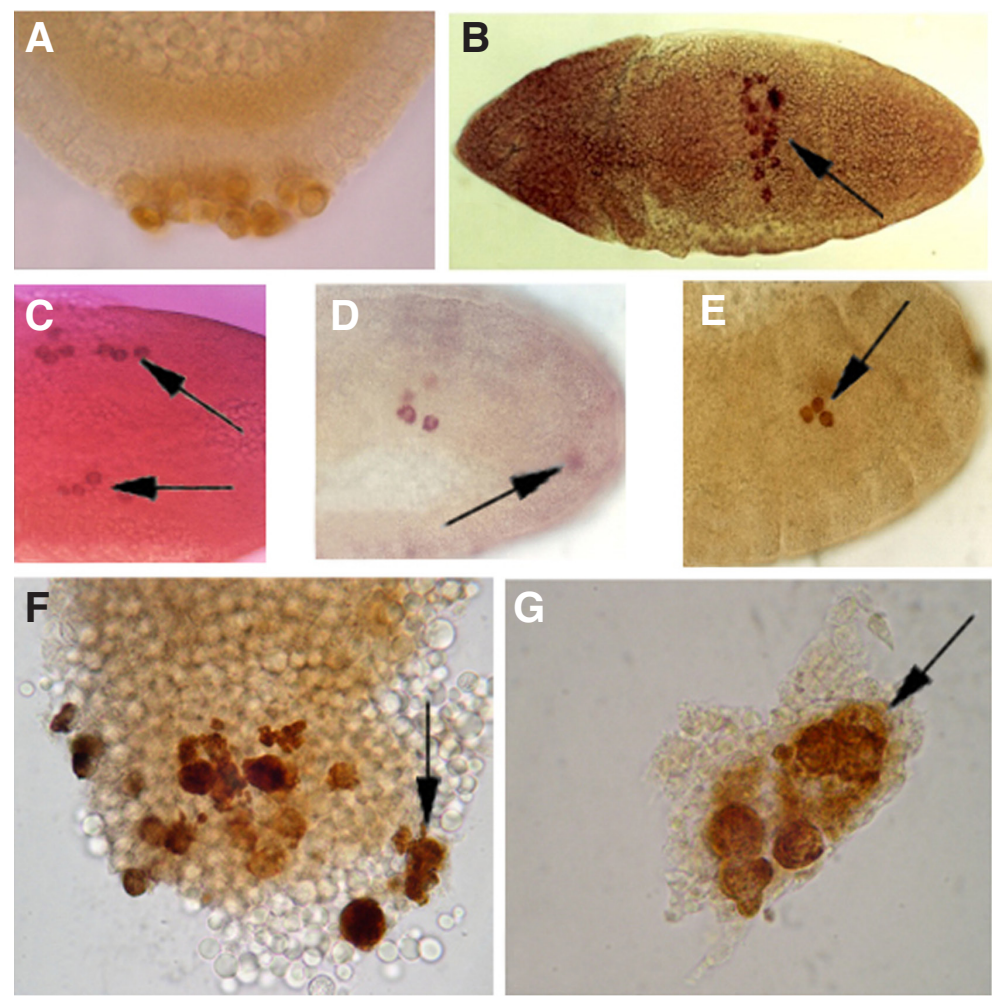

throughout development (Lasko and Ashburner, 1990; Styhler et al., 1998; Spradling et al., 2012). To determine the time of germline cell loss in $D$. virilis hybrids from dysgenic crosses, we compared the localization and the number of pole cells in embryos of parental strains and their hybrids at various stages. In our work, we explored conservative nature of the Vasa protein and using antibodies against D. melanogaster Vasa protein demonstrated that pole cell formation at the posterior pole of embryos from dysgenic crosses appeared normal. Thus, we usually observed 16-19 phenotypically normal round pole cells at the cellular blastoderm stage in embryos of the parental strains and dysgenic hybrids (Fig. 2 A), and the large processes/filopodial extensions usually produced by Drosophila pole cells at the early gastrula (Jaglarz and Howard, 1995) were seen at this stage in $D$. virilis strains and dysgenic hybrids (Supp. Fig. S2 A-C).

Next we monitored pole cell migration from the posterior pole through the midgut to the final location where germ cells form the primordial gonads (Fig. $2 \mathrm{~A}-\mathrm{E}$ ). Our observations indicate that in dysgenic embryos, although most pole cells did migrate into the gonads (Fig. $2 \mathrm{C}$ ), some pole cells cross the midgut epithelium but failed to reach the gonadal mesoderm (Fig. 2 D and E). Ultimately, these pole cells became mislocalized, lost their round shape, remain spread throughout the embryo and eventually died after migration through the posterior midgut wall (Fig. $2 \mathrm{~F}$ and G).

It has been previously demonstrated that apoptosis occurs in the primordial germ cells (PGCs) that leave the germline fate in response to genetic and environmental perturbations (Sano et al., 2002; Sato et al., 2007).

Overall, the migration defects in dysgenic embryos were observed starting from stage 12 .

Later, at the time of gonadal coalescence at stage 14, some pole cells had arrived at the gonad area, but the number of the cells forming the primary gonad was often reduced and varied from 3 to 15 in dysgenic embryos (Fig. 2C, Table 4). Sometimes, only one gonad in the dysgenic embryo was visible. The data summarized in Table 4 and Fig. 2 illustrate a variable penetrance of this phenotype.

\section{Manifestations of gonadal dysgenesis (GD) at the larval and pupal stages}

To determine whether germline cells die at postembryonic stages of development in dysgenic hybrids, we scored the germ cell number not only in the dysgenic embryos but also in the third instar larvae gonads. The data summarized in Table 4 indicate that the ratio of classes with different germline cells

Fig. 2. D. virilis dysgenic embryos stained with anti-Vasa antibodies.(A) Normal pole cells in strain 9 embryos at the stage of syncytial blastoderm; (B) Germline cells at the dorsal side of the embryo after they left midgut area (indicated by an arrow); (C) The final location of the germline cells forming severely reduced bilateral gonads indicated by arrows. (D,E) Dysgenic embryos at the various stages of pole cell migration to the location of primordial gonads possessing 3 to 15 pole cells. The arrows in D and E mark the mislocalized pole cells. (F) The dissected midgut area of dysgenic embryo at stage 10 at the moment, when pole cells are moving through the area. (G) The area of dissected dysgenic embryo (stage 14) at the moment of primordial gonad formation. The arrows indicate individual pole cells. $100 x$ oil immersion. The embryos in this figure are representative of $>50$ samples. 
quantity is similar in both stages. Therefore, the major germ cell loss likely occurs in the hybrid embryos at the stages indicated above, and the abnormal gonad development observed at the later stages is of a secondary nature. However, our analysis does not exclude a certain level of germline cell loss at later stages of dysgenic hybrid metamorphosis.

Thus, while complete unilateral atrophy was often observed in the dysgenic imagoes (Table 1) the formation of only one primordial gonad in dysgenic embryos represents an extremely rare phenomenon.

To further investigate the fate of germline cells in dysgenic hybrids, we stained isolated $D$. virilis dysgenic ovaries of third instar larvae with anti-Vasa antibodies. Sometimes dysgenic gonads exhibited apparently normal process of ovariole formation from germ cell proliferation to the formation of filaments of primordial ovarioles at the top of a gonad (Supp. Fig. S3 $A, B)$. In contrast, in most dysgenic larvae, the number of germ cells was strongly reduced (Supp. Fig. S3 C,D) and we often observed local concentration of germ cells at only one side of the primordial gonad (e.g. Supp. Fig. S3C). The same was true for the pupal ovaries (Supp. Fig. S4). Furthermore, in the ovaries of dysgenic larvae and late pupae stained with anti-Vasa antibodies, typical agametic and tumor-like ovarioles were often observed in the same ovary among other phenotypically normal ovarioles (Supp. Fig. S4).

Similarly, analysis of the male gonads demonstrated that in the testes of dysgenic larvae and pupae, the spermatid clusters frequently had abnormal structure (Supp. Fig. S5A-C) and were often severely reduced in number or altogether lacking (Supp. Fig. S5 D-F).

\section{Developmental expression and biogenesis of transposons in D. virilis strains and interstrain hybrids}

Previously, it has been demonstrated that several unrelated TEs, namely, Penelope, Ulysses, Paris, Telemac and Helena, are mobilized in $D$. virilis dysgenic hybrids (Scheinker et al., 1990; Petrov et al., 1995; Evgen'ev et al., 1997), and this mobilization likely leads to the multiple abnormalities observed in the progeny of dysgenic crosses. We also demonstrated using Northern hybridization that Penelopetranscription is significantly induced in the ovaries of the hybrids from dysgenic crosses (Evgen'ev et al., 1997). Here, we performed whole-mount hybridization experiments exploring probes homologous to Penelope and Ulysses, which are activated in $\mathrm{HD}$, and demonstrated that the detected levels of transcripts of both of these TEs were significantly higher in the ovaries of dysgenic females compared with the reciprocal hybrids (Fig. 3). These observations corroborated our previous conclusions based on Northern hybridization (Evgen'evet al., 1997). Interestingly, the signal in both cases was clearly observed in the cytoplasm of nurse cells (Fig. 3 B,D). We failed to observe any induction of gypsy transcription in the dysgenic ovaries (data not shown) and according to various data, gypsy in $D$. virilis is not activated by dysgenic crosses and does not transpose in $D$. virilis strains (Petrov et al., 1995, Rozhkov et al., 2011).

Characteristically, we did not detect Penelope transcripts in agametic ovarioles that probably resulted from the death of germline cells at the previous stages of development (Fig. 3B).

Next, we performed RT-PCR experiments to determine the stages when the transcription of several TEs including the ones
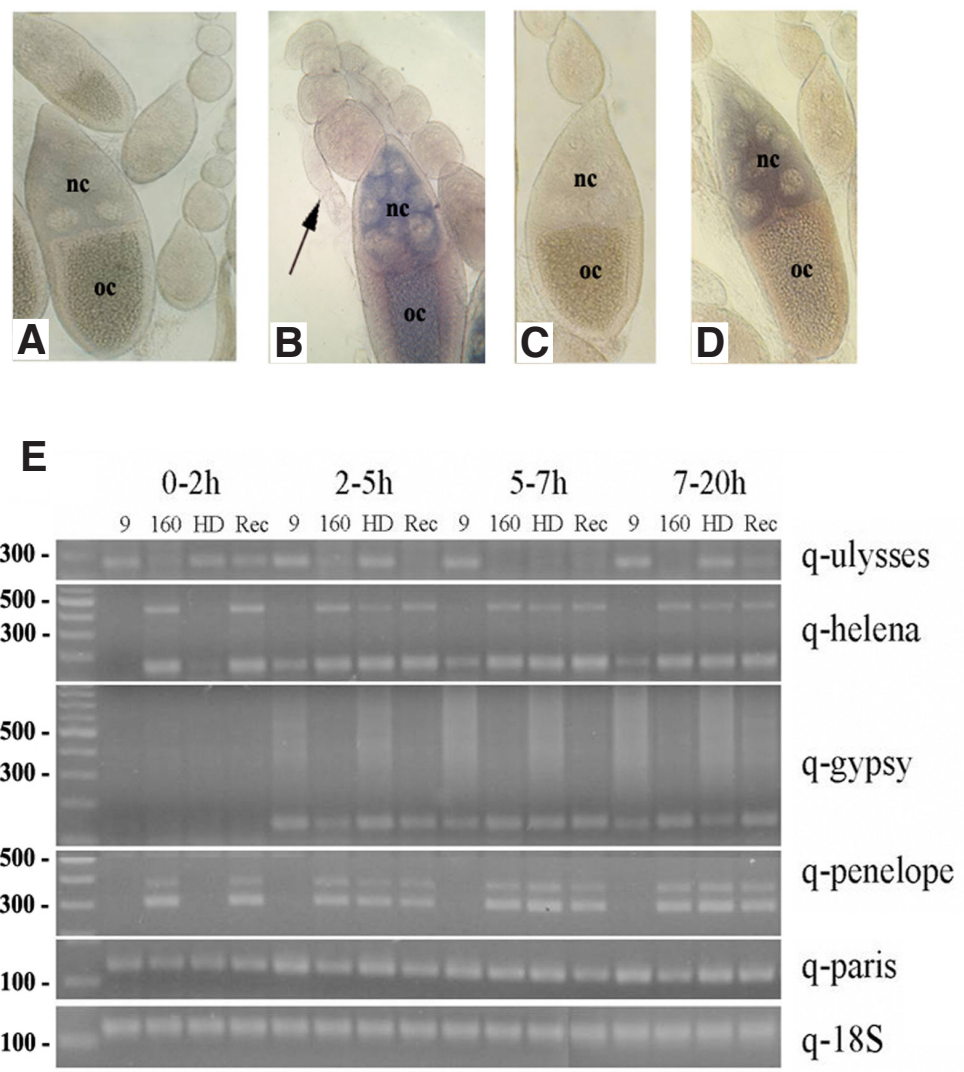

Fig. 3. Transcription levels of different transposons in the embryos of $D$. virilis strains and hybrids from reciprocal and dysgenic crosses. $(A, B)$ Whole mount hybridization with Penelope probe was performed to detect transcripts in the ovaries of $\mathrm{D}$. virilis reciprocal (A) and dysgenic (B) hybrids. An arrow indicates "agametic" ovariole from the same ovary with the normallooking ones, which shows only background staining with Penelope probe. (C,D) Whole-mount hybridization was performed with the Ulysses DNA probe; (C) reciprocal hybrids; (D) dysgenic hybrids; oc-oocyte, nc-nurse cells; 40x. (E) Semiquantative RT-PCR data for the transcription of five transposons at different embryonic stages of $\mathrm{D}$. virilis parental strains and interstrain hybrids. The two bands seen in Penelope gels apparently represent intronless and intronic Penelope-derived RNAs.

activated by dysgenic crosses begins. Fig. 3E shows that transcription of Penelope, Helena and Gypsy, had already begun in 2-5-hour embryos at the stage of the syncytial blastoderm. At least this statement can be safely made for the above three TEs that apparently do not have maternally transmitted transcripts (Fig. 3E).

The analysis performed indicated that RNAs homologous to all five TEs studied are present at all stages of embryogenesis following syncytial blastoderm in dysgenic and reciprocal hybrids.

It is known that full-length Penelope and Helena (Evgen'evet al., 1997; Vieira et al., 1998) are not present in the genome of $D$. virilis M-like strain 9, and hence, the piRNAs homologous to these TEs should not be maternally transmitted to the dysgenic embryos. The results depicted in Fig. 3E corroborated this notion because we failed to detect Penelope and Helena-derived transcripts in the early embryos (0-2h) of strain 9 and in dysgenic embryos at this stage. As for Paris we detected one euchromatic site (60B) of this TE in the microchromosome (chromosome 6 ) of $D$. virilis strain 9 which coincides with the previously obtained results where besides 
TABLE 3

THE FREQUENCY (\%) OF DIFFERENT TYPES OF TESTES IN THE
MALES OF STRAINS AND F1 CROSSES (FEMALES X MALES)

\begin{tabular}{cccccccc}
$\begin{array}{c}\text { Strains } \\
\text { and crosses }\end{array}$ & $\begin{array}{c}\text { No. of } \\
\text { males }\end{array}$ & $\mathbf{N} / \mathbf{N}$ & $\mathbf{N} / \mathbf{S N}$ & $\mathbf{N} / \mathbf{S T}$ & $\mathbf{S N / S N}$ & $\mathbf{S N} / \mathbf{S T}$ & $\mathbf{S T / S T}$ \\
\hline 9 & 125 & $100(125)$ & 0 & 0 & 0 & 0 & 0 \\
$9 \times 160$ & 357 & $49(173)$ & $11(38)$ & $16(57)$ & $3(11)$ & $5(17)$ & $16(58)$ \\
$160 \times 9$ & 100 & $99(99)$ & 0 & 0 & 0 & 0 & $1(1)$ \\
160 & 10010 & $93(93)$ & 0 & $5(5)$ & $1(1)$ & 0 & $1(1)$ \\
13 & 166 & $89(148)$ & 0 & $6(10)$ & $1(2)$ & 0 & $4(6)$ \\
$13 \times 160$ & 211 & $54(114)$ & $7(15)$ & $21(44)$ & $3(7)$ & $1(1)$ & $14(30)$ \\
$160 \times 13$ & 139 & $95(132)$ & 0 & $3(4)$ & $2(3)$ & 0 & 0 \\
1 & 32 & $100(32)$ & 0 & 0 & 0 & 0 & 0 \\
$1 \times 160$ & 54 & $100(54)$ & 0 & 0 & 0 & 0 & 0 \\
142 & 30 & $90(27)$ & 0 & 0 & 0 & 0 & $10(3)$ \\
$142 \times 160$ & 63 & $91(57)$ & 0 & $6(4)$ & 0 & 0 & $3(2)$ \\
$160 \times 142$ & 68 & $99(67)$ & $1(1)$ & 0 & 0 & 0 & 0 \\
110 & 39 & $97(38)$ & 0 & $3(1)$ & 0 & 0 & 0 \\
$110 \times 160$ & 129 & $96(124)$ & 0 & $2(3)$ & 0 & 0 & $2(2)$ \\
$160 \times 110$ & 77 & $94(72)$ & $1(1)$ & 0 & $4(3)$ & 0 & $1(1)$ \\
$142 \times 110$ & 45 & $100(45)$ & 0 & 0 & 0 & 0 & 0 \\
$110 \times 142$ & 56 & $98(55)$ & 0 & 0 & 0 & 0 & $2(1)$ \\
\hline
\end{tabular}

$\mathrm{N} / \mathrm{N}$ - both normal testis, N/SN - one normal and another semi-sterile (see text) testis, N/ST - normal/ sterile, SN/SN - both semi-sterile, SN/ST - semi-sterile/sterile, ST/ST - both sterile. The numbers in brackets indicate the number of males with different levels of sterility.

a few euchromatic sites of Paris and Helena several heterochromatic copies of these TEs were also detected (Petrov et al., 1995). Among these transposons, only gypsyDv showed somatic nature, since its transcripts were not detected in 0-2h zygotic embryos of both parents and their hybrids.

It was demonstrated (Blumenstiel and Hartl, 2005) that there is a correlation between maternally inherited Penelope-derived small RNAs and the sterility phenotypes observed in the adult $D$. virilis dysgenic hybrids. However, in this work the authors did not discriminate between Penelope-derived si- and piRNAs and besides small RNAs homologous to other TEs mobilized in HD were not monitored. Here we extended our analysis to other transposons and demonstrated (Fig. 4) that there is drastic difference between the levels of piRNAs homologous to Penelope, Helena and Paris determined in the embryos from reciprocal and dysgenic crosses. Dysgenic embryos were characterized by very low quantity of correspondent piRNAs (Fig. 4).

The trace amounts of Penelope and Paris-derived piRNAs observed in dysgenic embryos probably represent primary piRNAs, that may be produced by highly diverged heterochromatic copies of these TEs present in the maternal genome of strain 9 and representing the remnants of ancient invasions of $D$. virilis by these TEs (Petrov et al., 1996; Lyozin et al., 2001). It is of note that in general the levels of maternally transmitted piRNAs homologous to the analyzed TEs in early embryos differ by hundreds fold with Ulysses and gypsy Dv retroelements exhibiting the highest level

TABLE 4

THE FREQUENCY (\%) OF EMBRYONIC GONADS
AND LARVAL OVARIES IS SHOWN WITH DIFFERENT
NUMBERS OF GERM CELLS IN DYSGENIC HYBRIDS

\begin{tabular}{ccccc} 
Stage & \multicolumn{4}{c}{ Germ cells number and \% from the primordial gonads studied } \\
\hline Embryos & $<5$ cells & $6-8$ cells & $9-12$ cells & $>15$ cells \\
$(\mathrm{n}=67)$ & $25(37 \%)$ & $32(46 \%)$ & $9(13 \%)$ & $3(4.5 \%)$ \\
Third instar larvae & $2-6$ cells & $9-20$ cells & $30-50$ cells & 100 cells \\
$(\mathrm{n}=44)$ & $12(27 \%)$ & $19(42 \%)$ & $10(23 \%)$ & $3(7 \%)$ \\
\hline
\end{tabular}

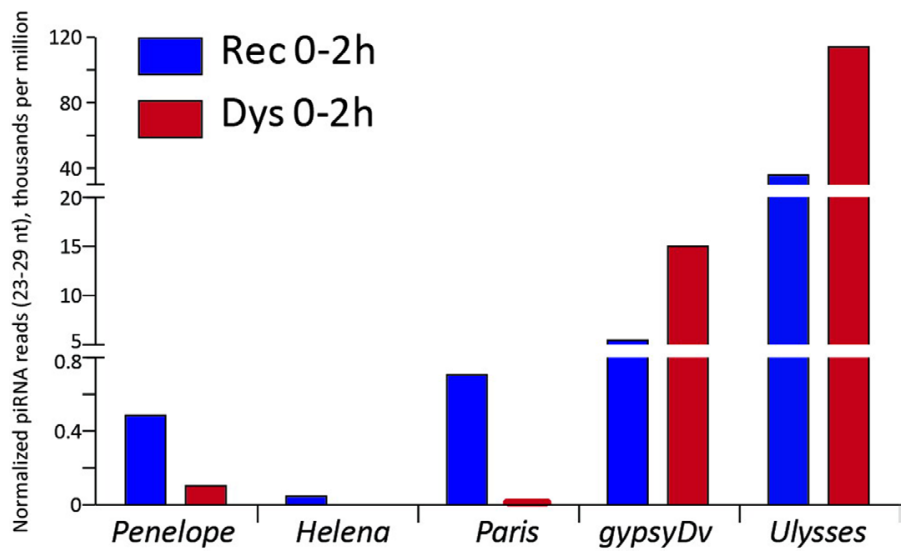

Fig. 4. Abundance of piRNA homologous to the five studied transposable elements (TEs) in early embryo libraries $(0-2 \mathrm{~h}$ ) of $D$. virilis reciprocal (in blue) and dysgenic (in red) hybrids. The characteristics of piRNAs homologous to the TEs were described previously (Rozhkov et al., 2010).

while Penelope and Helena showing comparatively low concentration of correspondent piRNAs (Fig. 4). However, the high level of maternal gypsyD $v$ in the 0-2h embryos does not match its presumable somatic transcription and, therefore, remains to be further investigated. Overall, these dramatic differences resemble different expression levels and/or biogenesis of TE-derived RNAs in the ovaries.

\section{Discussion}

During the period of HD investigation in D. virilis (1989-2012), the parental strains and particularly strain 160 underwent evident modifications. Thus, the number of Penelope copies in this strain as determined through in situ hybridization increased from 37 in 1989 to 54-55 in 2010 (Rozhkov et al., 2011). Furthermore, while we failed to observe any increase in gonadal sterility frequency under cold or high temperature conditions in 1989 (Lozovskaya et al., 1990), in 2010, the level of GS in dysgenic males and females, in contrast to such in $P-M \mathrm{HD}$, decreased with age of the parents involved in the crosses and increased when flies were grown under low temperature conditions (Tables S2 and S3). On the other hand in contrast to $P-M$ system in $D$. melanogaster we did not observe any restoration of fertility in the aged dysgenic hybrids with time (Khurana et al., 2011) and 7 day-old dysgenic flies exhibit the same level of sterility as 35 days hybrids (Table S2).

Phenotypic analysis of ovarioles in dysgenic hybrids allows to suggest the possible nature of the defects observed. Thus, agametic ovarioles in newly hatched females are the result of severe pole cell disturbances in embryos, while tumor-like ovarioles probably resulted from uncontrolled division of germ stem cells. The most frequent ovariole and/or follicle phenotypic abnormalities observed in $D$. virilis dysgenic hybrids and occasionally in individual strains may be summarized as the following: 1. "agametic"; 2 . "tumorlike"; 3. "beads-on-a-string" ovarioles with the chain of "dilatations" resulting from follicle degeneration, 4 . small oocyte enclosed by a chorion in an egg chamber; and 5. egg chamber with abnormal, reversed polarity. Notably, there are multiple well-known genes required for germ cell migration, that can mutate to induce various abnormalities in pole cell behavior or induce programmed death (Lasko, 1994; Deshpande et al., 2001; Coffman et al., 2002; Yatsu 
et al., 2008; Spradling et al., 2012). The disturbances in germ cell behavior observed in $D$. virilis dysgenic hybrids as well as in the $P$-M syndrome described in $D$. melanogaster are phenotypically but not necessary molecularly similar to abnormalities exhibited by the many known mutations that disrupt germ cell migration and development but never exhibit reciprocal effect in crosses.

In $D$. melanogaster $P-M$ and $D$. virilis HD systems, the germ cell abnormalities are observed when females lacking specific TEs are crossed with males carrying multiple copies of these TEs. Furthermore, in both systems, maximal levels of F1 sterility are temperature-dependent.

Characteristically, the most extreme sterile phenotype is agametic in both systems, while the less extreme phenotypes comprise a set of different classes, which were not quantitatively investigated in the $P-M$ system yet. While in $D$. melanogaster the activation of a single transposon, $P$-element, is responsible for the dysgenic phenotype, it has been suggested that in the case of $D$. virilis, the mobilization of several unrelated TEs is responsible for HD with Penelope possibly playing the pivotal role in the HD (Evgen'ev et al., 1997; Vieira et al., 1998; Blumenstiel and Hartl, 2005). However, it has recently been shown that several strains containing multiple intact Penelope copies behave as neutral and, hence, do not produce dysgenic phenotypes when crossed with $M$-like or $P$-like strains. Furthermore, there are a few atypical strains (e.g., 142) that do not contain full-length euchromaticPenelope copies but behave similar to weak $M$ - or neutral strains when crossed with strain 160 males (Tables 1 and 3). These results suggest that previously used strain classifications ( $P$-like, $M$-like and neutral) based on the presence of full-size Penelope copies (Evgen'ev et al., 1997; Vieira et al., 1998; Bluminstiel and Hartl, 2005) represent an oversimplification, and it is likely that other TEs, in addition to Penelope and unknown host genome factors, are also involved in determining the strain cytotype.

In the vast majority of HD studies, the analysis involved female and male gonad development and function in the dysgenic adult hybrids (Kidwell and Lisch, 2002; Bucheton et al., 2002). In contrast, here, we monitored the initial manifestations of the HD syndrome starting from early dysgenic embryos through the larval and pupal stages up to adults.

It is noteworthy that the analysis of the most extreme mutant sterile phenotypes in the $P-M$ system suggested that the defects predominantly arise at mid- to late embryonic and early larval development of F1 dysgenic hybrids, likely resulting from the $P$ element transposase activity leading to chromosome breakage "pulverization" and "hops" into multiple sites within a genome in PGCs (Kidwell and Novy, 1979; Rio, 2002; Kidwell and Lisch, 2002). It has also been demonstrated that retrotransposon mobilization induces DNA breaks and apoptosis in human cancer cells (Belgnaoui et al., 2006). Moreover, there are multiple mutations in $D$. melanogaster that lead to programmed death and/or various abnormalities of pole cell behavior (Lasko and Ashburner, 1990; Coffman et al., 2002; Spradling et al., 2012). In a few cases, the molecular mechanism of certain mutation action was determined. Thus, it was shown in D. melanogaster that pole cells lacking maternal NOS enter the apoptotic pathway and are unable to migrate properly into the embryonic gonads. It is believed that the failure to establish/maintain transcriptional quiescence in germ cells, which normally occurs in the precellular blastoderm, is likely to be the major cause of the migration defects in nos and pum embryos (Deshpande et al., 2001).

It has also been demonstrated that the apoptosis occurs in PGCs that leave the germline fate in response to genetic and environmental perturbations (Sato et al., 2007). Although we observe similar abnormalities in the behavior of pole cells, including their loss in the process of migration, in the early $D$. virilis dysgenic embryos, the molecular defects underlying this phenotype are unknown. It is clear, however, that the self-renewal of germline cells is disturbed in dysgenic hybrids because we observed high levels of gonad atrophy at all stages of fly development. We speculate that the abnormalities including germ cells death observed in early $D$. virilis dysgenic embryos may result from the expression of a few TEs (i.e., Penelope and Helena) at these stages in the absence of homologous maternally inherited primary piRNAs, which should provide silencing of TEs and, hence, inhibit their deleterious effects such as breakage of DNA in the early stages of ontogenesis (Brennecke et al., 2008; Rozhkov et al., 2010; Khurana et al., 2011).

Consistent with this idea, the defects in migration first become evident around the time the pole cells begin to exit the midgut, and at this stage, the transcription of all studied TEs, including Penelope and Helena, that are absent in the maternal genome (strain 9) already begins in dysgenic embryos (Fig. 4).

The expression of Penelope and other TEs at the absence of homologous silencing piRNA at early stages of embryogenesis in dysgenic hybrids might lead to the observed abnormalities in pole cell migration and death of a significant part of germline cells. The consequences of these germline disturbances may also be realized epigenetically at the later stages of ontogenesis, including adult dysgenic hybrids.

\section{Materials and Methods}

\section{Fly strains and crosses}

Three D. virilis wild strains, 9 (Batumi, Georgia), 13 (Krasnodar), and 1 (Erevan), and three marker strains $(160,142,110)$ were used in this study. In addition, $D$. melanogaster M-like strains ( $\left.D f(1), y w^{67 c 23(2)}\right)$, the Harwich strong $P$-like strain and the reciprocal hybrids between these strains were analyzed. All $D$. virilis strains used here have been previously described (Lozovskaya et al., 1990; Evgen'evet al., 1997; Vieira et al., 1998; Zelentsova et al., 1999). The flies were raised on standard media containing propionic acid as a mold inhibitor, with the addition of live yeast. The basic crosses (dysgenic crosses) involved males of the strain 160 (carrying approximately 53-54 Penelope copies) and females of wild-type or marker laboratory strains. As a control, reciprocal crosses and parental strains were used. For an estimation of initial fecundity (the number of all ovarioles in both ovaries), samples of 30 females from various strains and crosses were prepared. The counting included all phenotypes. To monitor gonadal atrophy at the level of individual ovarioles, we divided all flies into the following categories: "normal", when the gonad had less than a $25 \%$ reduction in size; "sterile", when the gonads were severely atrophied and apparently completely sterile; and "semi-normal", when the gonads were reduced in size by at least $25 \%$. We also distinguished between bilateral and unilateral atrophy.

\section{Gonad preparation and staining}

The flies were dissected in physiological solution $(0,9 \% \mathrm{NaCl})$ or PBS. For certain experiments, neutral red $(0.1 \%)$ was added into the saline solution to determine the levels of cell death. The ovaries and testes were extracted under a $2 \times 10$ magnification and dissected with fine needles. To protect the preparations from dehydration, they were typically covered with a drop of neutral paraffin oil. For ovariole counting and investigation, 
the preparations were used as "hanged-drops" and analyzed under the microscope under 20x, 40x, and 60x magnifications with phase contrast and 90x oil immersion. DAPI staining of the embryos was performed as previously described (Rothwell and Sullivan, 2007).

\section{Whole mount hybridization with Penelope and Ulysses probes}

DIG labeling and detection of the transposon DNA probes used in the in situ hybridization experiments were performed as previously described (Roche 2006; Rozhkov et al., 2011).

Ovaries of 5-7-day-old dysgenic and reciprocal females were dissected in PBS and fixed with a 4\% paraformaldehyde/PBS solution for 20 min at RT. The treatment of ovaries with a $20 \mu \mathrm{g} / \mathrm{ml}$ proteinase K/PBS solution for 15 min was followed by fixation in a $4 \%$ paraformaldehyde/PBS solution for 20 min at RT. During these steps, PBS with $0.1 \%$ Tween-20 (PBT) was used as a rinsing solution. Pre-hybridization and hybridization steps were performed at $60^{\circ} \mathrm{C}$ in hybridization buffer ( $50 \%$ formamide, $5 x$ SSC, $0.1 \%$ Tween $20,1 \mathrm{mg} / \mathrm{ml}$ tRNA and $50 \mu \mathrm{g} / \mathrm{ml}$ heparin). The anti-DIG-AP antibody (Roche) was used at a 1:2000 dilution. The color typically developed within one hour.

\section{Vasa- and 1B1-antibody staining}

We utilized a previously described method (Styhler et al., 1998) with minor modifications. Briefly, the embryos of the $D$. virilis parental strains (9 and 160) and hybrids were collected on agarose plates for 24 hours, washed in $\mathrm{H}_{2} \mathrm{O}$ and dechlorinated in $5 \%$ hypochloride. Then, the embryos were washed with $\mathrm{H}_{2} \mathrm{O}$ and fixed in heptane saturated with $4 \%$ paraformaldehyde for $20 \mathrm{~min}$ on a rolling platform. The embryos were devitellinized by shaking in absolute methanol and stored at $-20^{\circ} \mathrm{C}$. The embryos and the fixed gonads dissected from the larvae and pupae were rehydrated in a methanol/PBS series, and, after PBS washes and preincubation in $1 \% B S A$, incubated overnight with rabbit anti-Vasa (1:1000) and mouse anti-1B1(1:50). The washes were performed with PBS for 1-2 hours with at least three solution changes. The secondary antibodies were biotinylated goat anti-rabbit lgG (1:2000) and goat anti-mouse $\lg G(1: 2000)$. The signal was amplified by incubation with preformed avidin-biotin complex according to the $A B C$ method (Vector Laboratories). The signal was detected by $\mathrm{DAB} /$ hydrogen peroxidase reaction. The Vasa-antibodies were kind gift of Dr. S. Styler from P.Lasko laboratory (McGill University, Canada) and 1B1 antibodies (against hu-li-tai-shao protein) were generously provided by Dr. N. Tulina from Carnegie Institute of Washington.

\section{Semiquantitative reverse transcription analysis (RT-PCR)}

The analyses were performed using $1 \mu \mathrm{g}$ of DNase I (Fermentas)-treated total RNA from different stage embryos. cDNAwas prepared using the Strand cDNA Synthesis Kit and random hexamer primers (Fermentas). A volume of $2 \mu \mathrm{l}$ of the 5 -fold diluted cDNA was used in the $30 \mu \mathrm{l}$ Taq-polymerase PCR mix (SibEnzyme) with 35 amplification cycles. All primers used in the investigation are given in Table S1.

\section{Small RNA library development from early embryos and analysis of transposon-derived RNAs}

The small RNA libraries from early embryos were cloned and analysed as described (Rozhkov et al., 2010, Rozhkov et al., 2011). Small RNAs were mapped to the latest releases of $D$. virilis genome. Transposon-derived small RNAs with up to three mismatches were mapped onto Repbase. For the transposon analysis, only small RNAs matching the bodies of selected five TEs were considered as the true TE-derived sequences. Small RNA counts were normalized to 1 million small RNAs after subtraction of small RNA reads corresponding to abundant noncoding RNAs.

\section{Acknowledgements}

This work was supported by the Russian Foundation for Basic Research, projects $N^{\circ}$ 09-04-00643 and 09-04-00660, project from "Genofond dynamics" program, and Grant of the Program of Molecular and Cellular Biology RAN to M.E. We are grateful to Dr. Sylvia Styhler (McGill University, Canada) and Dr. N. Tulina (Carnegie Institute of Washington) for the kind gift of the rabbit Vasa-antibody and $1 B 1$ antibodies, respectively.

\section{References}

ARKHIPOVA, I.R, PYATKOV, K.I., MESELSON, M. and EVGEN'EV, M.B. (2003). Retroelements containing introns in diverse invertebrate taxa. Nat Genet33: 123-124

BLUMENSTIEL, J.P. and HARTL D.L. (2005).Evidence for maternally transmitted small interfering RNA in the repression of transposition in Drosophila virilis. Proc Natl Acad Sci USA 102: 15965-15970.

BRENNECKE, J., MALONE, C.D., ARAVIN, A.A., SACHIDANANDAM, R., STARK, A. and HANNON, G.J. (2008). An epigenetic role for maternally inherited piRNAs in transposon silencing. Science 322: 1387-1392.

BUCHETON, A., BUSSEAU, I. and TENINGES, D. (2002). I elements in Drosophila melanogaster.Mobile DNA II. (Craig, N.L., Craigie, R., Gellert, M., Lambowitz, A.M. eds.). Washington, DC. ASM Press, pp. 796-812.

BELGNAOUI, S.M., GOSDEN, R.G., SEMMES, O.J. AND HAOUDI, A. (2006) Human LINE-1 retrotransposon induces DNA damage and apoptosis in cancer cells. Cancer Cell Int. 6: 13.

CAMPOS-ORTEGA, J.A. and HARTENSTEIN, J. (1985). The embryonic development of $D$. melanogaster. Springer-Verlag, Berlin.

CHAMBEYRON, S., POPKOVA, A., PAYEN-GROSCHÊNE, G., BRUN, C., LAOUINI, D., PELISSON, A. and BUCHETON, A. (2008).piRNA mediated nuclear accumulation of retrotransposon transcripts in the Drosophila female germline. Proc Nat Acad Sci USA 105: 14964-14969.

COFFMAN, R.C., STROHM, R.C., OAKLEY, F.D.,YAMADA, Y., PRZYCHODZIN, D., and BOSWELL, R.E. (2002). Identification of X-linked genes required for migration and programmed cell death of Drosophila melanogaster germ cells. Genetics 162: 273-284.

DESHPANDE, G., SWANHART, L., CHIANG, P. and SCHEDL, P. (2001). Hedgehog signaling in germ cell migration. Cell 106: 759-769.

EVGEN'EV, M.B., ZELENTSOVA, H., SHOSTAK, N., KOZITSINA, M., BARSKYI, V. LANKENAU, D.H. and CORCES, V.G. (1997).Penelope, a new family of transposable elements and its possible role in hybrid dysgenesis in Drosophila virilis. Proc Natl Acad Sci USA 94: 196-201.

FINNEGAN, D.J. (1989). The I factor and I-R hybrid dysgenesis in Drosophila melanogaster. Mobile DNA. (Berg, D.E. and Howe M.M. eds.). Washington, DC. American Society for microbiology pp. 503-517.

HUYNH, J.R. (2005). Fusome as a Cell-Cell Communication Channel of Drosophila Ovarian Cyst. In Cell-Cell Channels. (F. Baluska, D. Volkman, P. Barlow., eds.) Eurecah.com.

JAGLARZ, M.K. and HOWARD, K.D. (1995). The active migration of Drosophila primordial germ cells. Development 121: 3495-3503.

KHURANA, J.S., WANG, J., XU, J., KOPPETSCH, B.S., THOMSON, T.C., NOWOSIELSKA, A., LI, C., ZAMORE, P.D., WENG, Z. and THEURKAUF, W.E. (2011). Adaptation to Pelement Transposon Invasion in Drosophila melanogaster. Cell 47: 1551-1563.

KIDWELL, M.G. (1983). Evolution of hybrid dysgenesis determinants in Drosophila melanogaster. Proc Natl Acad Sci USA 80: 1655-1659.

KIDWELL, M.G. and LISCH, D.R. (2002). Transposable elements as sources of genomic variation. Mobile DNA II. (Craig, N.L., Craigie, R., Gellert, M., Lambowitz, A.M. eds). Washington, DC. ASM Press pp. 796-812

LANGE, A.B. and CHYONG KUANG KHOK. (1981). Abortive oogenesis and the physiological age of blood-sucking mosquitoes (Diptera, Culicidae). Med Parazitol (Mosk) 50: 48-56.

LASKO, P.F. and ASHBURNER, M. (1990). Posterior localization of vasa protein correlates with, but is not sufficient for pole cell development. Genes Dev 4: 905-921

LASKI, F.A. and RUBIN, G.M. (1989). Analysis of the cis-acting requirements for germline-specific splicing of the P-element ORF2-ORF3 intron. Genes Dev 3: 720-728.

LOZOVSKAYA, E.R., SCHEINKER, V.S. and EVGEN'EV, M.B. (1990). A hybrid dysgenesis syndrome in Drosophila virilis. Genetics 126: 619-623.

LYOZIN, G.T., MAKAROVA, K.S., VELIKODVORSKAYA, V.V., ZELENTSOVA, E.S. KHECHUMIAN, R.R., KIDWELL, M.G., KOONIN, E.V. and EVGEN'EV, M.B. (2001) The structure and evolution of Penelope in the virilis species group of Drosophila: an ancient lineage of retroelements. J Mol Evol 52: 445-456. 
MATHE, A. (2004). Immunocytological analysis of oogenesis. Drosophila Cytogenetic Protocols. (Henderson, D.S. ed.). Human Press pp. 89-128.

NIKI, Y. (1986) Germline autonomous sterility of P-M dysgenic hybrids and their application to germline transfers in Drosophila melanogaster. Dev Biol 113: 255-258.

PETROV, D.A., SCHUTZMAN, J.L., HARTL, D.L. and LOZOVSKAYA, E.R. (1995). Diverse transposable elements are mobilized in hybrid dysgenesis in Drosophila virilis. Proc Natl Acad Sci USA 92: 8050-8054.

RIO, D.C. (2002). P transposable elements in Drosophila melanogaster. Mobile DNA II. (Craig, N.L., Craigie, R., Gellert, M., Lambowitz, A.M. eds). Washington, DC. ASM Press pp. 796-812.

ROZHKOV, N.V., ARAVIN, A.A., ZELENTSOVA, E.S., SCHOSTAK, N.G., SACHIDANANDAM, R., MCCOMBIE, W.R., HANNON, G.J. and EVGEN'EV, M.B. (2010). Small RNA-based silencing strategies for transposons in the process of invading Drosophila species. RNA 16: 1634-1645.

ROZHKOV, N.V., ZELENTSOVA, E.S., SHOSTAK, N.G. and EVGEN'EV, M.B. (2011). Expression of Drosophila virilis retroelements and role of small RNAs in their intrastrain transposition. PLoS One 6: e21883.

ROCHE, P.J. (2006). Preparation of template DNA and labeling techniques. In situ Hybridization Protocols. (Darby IA, Hewitson TD eds). Humana Press. pp. 9-16.

ROTHWELL W.F., and SULLIVAN, W. (2007). DAPI Staining of Drosophila Embryos. CSH Protoc. 2007 pdb.prot4834.

SANO, H., NAKAMURA, A. and KOBAYASHI, S. (2002). Identification of a transcriptional regulatory region for germline-specific expression of vasa gene in Drosophila melanogaster. Mech Dev 112: 129-139.

SATO, K., HAYASHI, Y., NINOMIYA, Y., SHIGENOBU, S., ARITA, K., MUKAI, M. and KOBAYASHI, S. (2007). Maternal Nanos represses hid/skl-dependent apoptosis to maintain the germ line in Drosophila embryos. Proc Natl Acad Sci USA 104: 7455-7460

SCHEINKER, V.S., LOZOVSKAYA, E.R., BISHOP, J.G., CORCES, V.G. and
EVGEN'EV, M.B. (1990). A long terminal repeat-containing retrotransposon is mobilized during hybrid dysgenesis in Drosophila virilis. Proc Natl Acad Sci USA 87: 9615-9619.

SCHOSTAK, N., PYATKOV, K., ZELENTSOVA, E., ARKHIPOVA, I., SHAGIN, D., SHAGINA, I., MUDRIK, E., BLINTSOV, A., CLARK, I., FINNEGAN, D.J. and EVGEN'EV, M.B. (2008). Molecular dissection of Penelope transposable element regulatory machinery. Nucleic Acids Res 36: 2522-2529.

SPICER, G. and BELL, C. (2002). Molecular phylogeny of the Drosophila virilis species group (Diptera: Drosophilidae) inferred from mitochondrial $12 \mathrm{~S}$ and $16 \mathrm{~S}$ ribosomal RNA genes. Ann Entomol Soc Am 95: 156-161.

SPRADLING, A.S. (1993). Developmental genetics of oogenesis. The development of Drosophila melanogaster. (Bate M., Arias A.M. eds). Cold Spring Harbor: Cold Spring Harbor Laboratory Press pp. 1-70.

SPRADLING,A., FULLER, M.T., BRAUN, R.E., and YOSHIDA, S. (2012). Germline stem cells. Cold Spring Harb Perspect Biol 3: a002642.

STYHLER, S., NAKAMURA, A., SWAN, A., SUTER, B., and LASKO, P. (1998). Vasa is required for GURKEN accumulation in the oocyte, and is involved in oocyte differentiation and germline cyst development. Development 125: 1569-1578.

VERHEYER,E., and COOLEY, L. (1994). Chapter 28 Looking at Oogenesis. Methods Cell Biol. 44: 545-561.

VIEIRA, J., VIEIRA, C.P., HARTL, D.L. and LOZOVSKAYA, E.R. (1998). Factors contributing to the hybrid dysgenesis syndrome in Drosophila virilis. Genet Res 71: 109-117.

YATSU, J., HAYASHI, M., MUKAI, M., ARITA, K., SHIGENOBU, S. and KOBAYASHI, S. (2008). Maternal RNAs encoding transcription factors for germline-specific gene expression in Drosophila embryos. Int J Dev Biol 52: 913-923.

ZELENTSOVA, H., POLUECTOVA, H., MNJOIAN, L., LYOZIN, G., VELEIKODVORSKAJA, V., ZHIVOTOVSKY, L., KIDWELL, M.G. and EVGEN'EV, M.B. (1999). Distribution and evolution of mobile elements in the virilis species group of Drosophila. Chromosoma 108: 443-456. 


\section{Further Related Reading, published previously in the Int. J. Dev. Biol.}

Recent advances in Drosophila stem cell biology

John Pearson, Lourdes López-Onieva, Patricia Rojas-Ríos and Acaimo González-Reyes

Int. J. Dev. Biol. (2009) 53: 1329-1339

http://dx.doi.org/10.1387/ijdb.072431.jp

mgm 1, the earliest sex-specific germline marker in Drosophila, reflects expression of the gene esg in male stem cells Adrian Streit, Luca Bernasconi, Pavel Sergeev, Alex Cruz and Monica Steinmann-Zwicky

Int. J. Dev. Biol. (2002) 46: 159-166

http://dx.doi.org/10.1387/ijdb.11902678

http://www.intjdevbiol.com/web/paper/11902678

Female sterile mutations and egg chamber development in Drosophila Melanogaster S Gigliotti, D Rotoli, A Manzi, F Graziani and C Malva

Int. J. Dev. Biol. (2000) 44: 581-589

http://www.intjdevbiol.com/web/paper/11061421

\section{Creating mosaics in Drosophila}

N Perrimon

Int. J. Dev. Biol. (1998) 42: 243-247

http://www.intjdevbiol.com/web/paper/9654004

The Drosophila Stock Centers and their implications for developmental biology

A Rasmuson-Lestander

Int. J. Dev. Biol. (1995) 39: 765-768

http://www.intjdevbiol.com/web/paper/8645560

Germ-line expression of a functional LINE from Drosophila Melanogaster: fine characterization allows for potential investigations of trans-regulators C Tatout, M Docquier, $\mathrm{P}$ Lachaume, M Mesure, $\mathrm{P}$ Lécher and $\mathrm{H}$ Pinon Int. J. Dev. Biol. (1994) 38: 27-33

http://www.intjdevbiol.com/web/paper/8074994

5 yr ISI Impact Factor $(2011)=2.959$
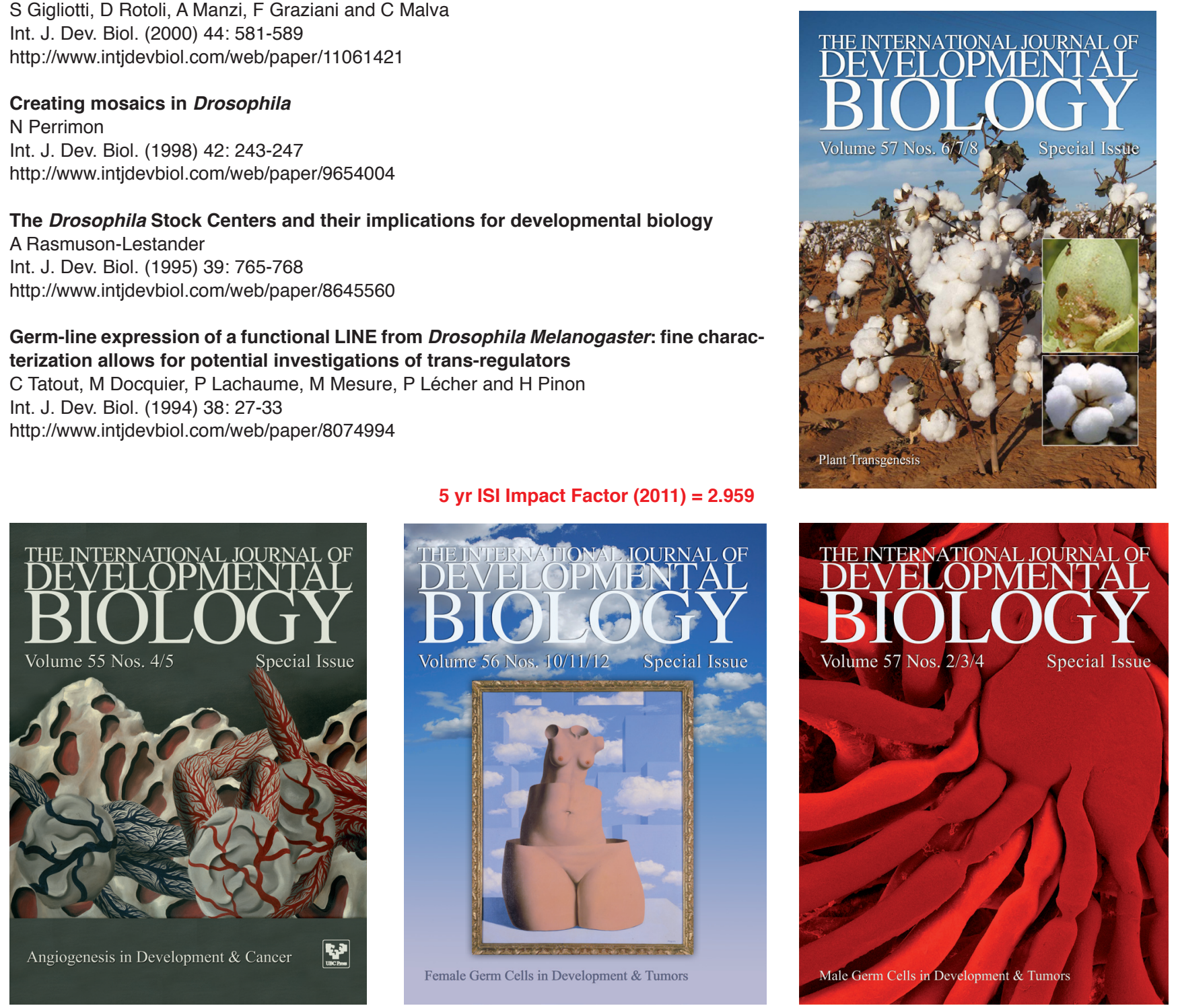